\title{
Joint Spatial-Temporal Spectrum Sensing for Cognitive Radio Networks
}

\author{
Tuan Do, Student Member, IEEE and Brian L. Mark Senior Member, IEEE
}

\begin{abstract}
In a wireless system with opportunistic spectrum sharing, secondary users equipped with cognitive radios attempt to access radio spectrum that is not being used by the primary licensed users. On a given frequency channel, a secondary user can perform spectrum sensing to determine spatial or temporal opportunities for spectrum reuse. Whereas most prior works address either spatial or temporal sensing in isolation, we propose a joint spatial-temporal spectrum sensing scheme, which exploits information from spatial sensing to improve the performance of temporal sensing. We quantify the performance benefit of the joint spatial-temporal scheme over pure spatial sensing and pure temporal sensing based on counting rule and linear quadratic detectors. Finally, we analyze a multi-level quantization feedback scheme that can improve the performance of temporal sensing based on counting rule detectors.
\end{abstract}

Index Terms-Dynamic spectrum access, cognitive radio, hypothesis testing, spectrum sensing

\section{INTRODUCTION}

In traditional wireless systems, spectrum or frequency is allocated to licensed users over a geographic area. Within these constraints, spectrum is considered a scarce resource due to static spectrum allocation. Recent empirical studies of radio spectrum usage have shown that licensed spectrum is typically highly under-utilized [2], [3]. To recapture the so-called "spectrum holes," various schemes for allowing unlicensed or secondary users to opportunistically access unused spectrum have been proposed. Opportunistic or dynamic spectrum access is achieved by cognitive radios that are capable of sensing the radio environment for spectrum holes and dynamically tuning to different frequency channels to access them. Such radios are often called frequency-agile or spectrum-agile.

On a given frequency channel, a spectrum hole can be characterized as spatial or temporal. A spatial spectrum hole can be specified in terms of the maximum transmission power that a secondary user can employ without causing harmful interference to primary users that are receiving transmissions from another primary user that is transmitting on the given channel. Spectrum reuse in this context is similar to frequency reuse among cochannel cells in a cellular network. A temporal spectrum hole is a period of time for which the primary

Copyright (c) 2010 IEEE. Personal use of this material is permitted. However, permission to use this material for any other purposes must be obtained from the IEEE by sending a request to pubs-permissions@ieee.org.

Manuscript received November 06, 2009; revised February 24, 2010; accepted April 11, 2010. The editor coordinating the review of this paper and approving it for publication was J. Deng. This work was supported in part by the U.S. National Science Foundation under Grants CNS-0520151 and ECS-0426925. An early version was presented in part at the Conference on Information Science and Systems (CISS) Mar. 2009 [1].

T. Do and B. L. Mark are with the Dept. of Electrical and Computer Engineering, George Mason University, Fairfax, VA 22030 (e-mail: tdoa@gmu.edu, bmark@gmu.edu). transmitter is idle. During such idle periods, a secondary user may opportunistically transmit on the given channel without causing harmful interference.

Spatial spectrum sensing is investigated [4], [5], wherein the maximum interference-free transmit power (MIFTP) of a given secondary user is estimated based on signal strengths received by a group of secondary nodes. To calculate the MIFTP for a secondary node, estimates of both the location and transmit power of the primary transmitter are estimated collaboratively by a group of secondary nodes. Using these estimates, each secondary node determines its approximate MIFTP, which bounds the size of its spatial spectrum hole. In [4], [5], the primary transmitters are assumed to transmit at constant powers. However, this assumption does not allow secondary users to take advantage of temporal spectrum holes. In practice, the primary transmitter may alternate between being active $(\mathrm{ON})$ and idle $(\mathrm{OFF})$.

The problem of detecting when the primary is ON or OFF is called temporal spectrum sensing. Cooperative temporal sensing has been studied in [6]-[8]. The decision on the ON/OFF status of the primary transmitter can be made either at individual secondary nodes or collaboratively by a group of secondary nodes. Cooperation among secondary nodes for temporal sensing can overcome problems posed by low signalto-noise ratio (SNR), shadowing, and hidden terminals [8]. A practical solution for cooperative temporal sensing is proposed in [8], whereby individual secondary nodes make decisions about the ON/OFF status of the primary transmitter independently. A fusion center or centralized controller collects the individual hard decisions made by all secondary nodes and then makes a final decision on whether the primary is idle or active. The fusion center is assumed to know the geographic locations of all cooperating secondary nodes and hence can estimate the correlations between their observations. However, the fusion center does not generally have knowledge of the primary's location or transmit power. A suboptimal temporal detector is proposed in [9] based on a linear quadratic (LQ) detector that uses partial statistical knowledge to improve detection performance. As discussed in [8], the LQ detector outperforms a simpler detector based on a counting rule in the regime of moderate to high correlation among the secondary nodes.

In this paper, we propose a joint spatial-temporal sensing scheme for wireless networks with opportunistic spectrum sharing. We consider the case of a single primary transmitter that alternates between ON and OFF states. During the ON state, secondary nodes perform collaborative spatial spectrum sensing. When the primary transmitter is in the ON state, the secondary nodes employ spatial spectrum sensing to esti- 
mate the MIFTP (cf. [4]). Estimation of the MIFTP involves localization of the primary transmitter and estimation of its transmit power. When the primary transmitter is in the OFF state, a given secondary user can transmit at maximum power. Here, spatial spectrum sensing relies on temporal spectrum sensing in order to determine the ON/OFF state of the primary transmitter. In a pure spatial sensing scheme, the primary transmitter is assumed to be $\mathrm{ON}$ at all times. Thus, when the primary is actually OFF for some portion of time, pure spatial sensing will tend to underestimate the transmit power of the primary. Temporal sensing information can be used to trigger spatial sensing activity only during the ON periods of the primary transmitter. This will result in a more accurate estimate of the primary transmitter parameters and hence improve the accuracy of spatial sensing.

Conversely, localization information for the primary transmitter obtained from spatial spectrum sensing are used to improve the performance of temporal sensing. Approximate knowledge of the primary transmitter's location are used to intelligently select a subset of the observations from secondary nodes for temporal sensing. Temporal sensing performance can be improved in this way because the observation set can be selected from the secondary nodes so as to minimize the correlations among the observations. Our simulation results show that the proposed spatial-temporal sensing scheme outperforms pure temporal sensing based on either a counting rule or LQ detector.

We also investigate a multi-level quantization detection strategy for temporal sensing based on the counting rule in which each secondary node sends an $m$-bit decision to the fusion center. Thus, the observations received from the secondary nodes are quantized to $2^{m}$ levels. Previous works on temporal spectrum sensing (cf. [6]-[8]) assume that each secondary node sends only a one-bit decision to the fusion center where the final decision is made on whether the primary is ON or OFF. This approach can be useful when there is very limited communication bandwidth between secondary nodes and fusion center, but it leads to significantly poorer performance compared to a centralized approach. A centralized fusion center computes the joint likelihood of all soft observations to obtain the final detection decision. However, the centralized approach is difficult to implement in practice because it requires a relatively large communication bandwidth between the secondary users and the fusion center. Therefore, the proposed multi-level feedback scheme represents a compromise between the distributed one-bit feedback scheme and the centralized detector.

The remainder of the paper is organized as follows. Section II describes the system model for spatial spectrum and temporal spectrum sensing. Section III develops that joint spatial-temporal sensing scheme and compares its achievable capacity relative to pure spatial and pure temporal sensing schemes. Section IV investigates the performance of temporal sensing based on the counting rule with multi-level feedback. Section V presents simulation results. Finally, the paper is concluded in Section VI.

\section{SySTEM ModeL}

We consider a discrete-time system model with a single primary transmitter and $M$ secondary users equipped with frequency-agile cognitive radios. The primary transmitter can be in one of two states: an ON state in which it transmits with constant power $s_{p}$, and an OFF state in which it does not transmit.

\section{A. Spatial Spectrum Sensing}

All transmissions are assumed to be omnidirectional and the signal propagation follows a lognormal shadowing model. We assume the following path loss model (cf. [10]):

$$
L=10 n \log _{10}\left(d / d_{0}\right)+L_{0}[\mathrm{~dB}],
$$

where $d$ is the distance between transmitting and receiving antennas in meters, $L$ is the path loss in $\mathrm{dB}, L_{0}$ is the attenuation at a reference distance $d_{0}, L_{0}=20 \log _{10}\left(\frac{4 \pi}{\lambda}\right)$ and $\lambda$ is the wavelength in meters. Accounting for the effect of shadowing and noise, the received power at node $v$ due to node $p$ can be represented as a lognormal random variable:

$$
R_{v}=s_{p}-10 n \log _{10}\left(d_{p, v} / d_{0}\right)+W[\mathrm{dBm}],
$$

where $n$ is the path loss factor, $s_{p}(\mathrm{dBm})$ is the transmit power of node $p$ at $d_{0}$, and $d_{i, j}$ denotes the distance between node $i$ and node $j$ in meters. Here, we approximate the sum of the shadowing and noise powers as a lognormally distributed random variable $W \sim \mathcal{N}\left(0, \sigma_{W}^{2}\right)$, where $\sigma_{W}^{2}$ is the shadowing noise variance. We define the path loss function $g(d) \triangleq 10 n \log _{10}\left(d / d_{0}\right)$. Then the path loss from node $i$ to node $j$ is given by

$$
L_{i, j} \triangleq g\left(d_{i, j}, n\right)+W[\mathrm{dBm}] .
$$

We shall make use of some concepts related to spatial spectrum sensing from [4]. The maximum interference-free transmit power (MIFTP) of a secondary node is defined as the maximum transmit power on a given channel such that the probability of interference to any potential victim node (i.e., a primary receiver) is less than a prescribed threshold. The outage probability of a victim node $v$ with respect to the transmitter $p$, is the probability that the received power $R_{v}$ from node $p$ falls below a predetermined detection threshold $r_{\text {min }}: P_{\text {out }}(p, v) \triangleq P\left(R_{v}<R_{\text {min }}\right)$. The coverage distance is the maximum distance between node $p$ and any potential victim node $v$ such that $P_{\text {out }}$ does not exceed a predefined threshold $\epsilon_{\text {cov }}>0: d_{\text {cov }}(p) \triangleq \max \left\{d_{p, v}: P_{\text {out }}(p, v) \leq \epsilon_{\text {cov }}\right\}$. The coverage area of the transmitter $p$ is the disk centered at node $p$ with radius $d_{\text {cov }}(p)$.

The received power at node $v$ from node $a$ is given by $I_{v}=s_{a}-g\left(d_{a, v}\right)+W$, where $s_{a}$ is the transmit power of $a$. The interference probability in the spatial domain with respect to a given victim node $v$ is the probability that $I_{v}$ exceeds a predefined interference tolerance threshold $i_{\max }: P_{s}(a, v) \triangleq$ $P\left(I_{v} \geq i_{\max }\right)$. For a single fixed primary transmitter $p$ and FAR node $a$, the MIFTP is the maximum transmit power of the FAR node such that the interference probability with respect 
to any potential victim node within the coverage distance from node $p$ does not exceed a threshold $\epsilon_{\text {int }}>0$ :

$$
s_{a}^{*} \triangleq \max \left\{s_{a}: P_{s}(a, v) \leq \epsilon_{\mathrm{int}}, \forall v: d_{p, v} \leq d_{\mathrm{cov}}(p)\right\} .
$$

The worst-case interference probability is given by

$$
P_{s}(a) \triangleq \max _{v} P_{s}(a, v)=Q\left(\frac{i_{\max }-s_{a}+g\left(d_{a}^{*}\right)}{\sigma_{W}}\right) .
$$

where $d_{a}^{*} \triangleq d_{p, a}-d_{\text {cov }}(p)$. An approximation for the MIFTP based on received signal strength measurements is developed for the case of a single primary transmitter in [4] and the case of multiple cochannel primary transmitters in [11], respectively.

To mitigate the effect of shadowing and low SNR, cooperation among the secondary nodes is necessary to perform both spatial and temporal spectrum sensing. We assume that all secondary nodes have the same detection distance, i.e., they are equipped with detectors having the same receiver sensitivity. The set of secondary nodes that performs temporal sensing may be different from the set of nodes that performs spatial sensing. Let $\mathcal{S}$ and $\mathcal{T}$ denote the sets of nodes that are involved in spatial sensing and temporal sensing, respectively. The nodes in $\mathcal{S}$ are assumed to be located within a circle centered at primary transmitter location $\left(x_{p}, y_{p}\right)$ with radius equal to the detection distance $d_{\operatorname{det}}(a)$.

\section{B. Temporal Sensing Model}

We adopt a model of temporal spectrum sensing similar to the one described in [8]. Each node in $\mathcal{T}$ makes an independent decision about the ON/OFF state of the primary transmitter. The fusion center randomly selects a subset $\tilde{\mathcal{T}} \subset \mathcal{T}$ of nodes and requests the ON/OFF decisions from the set of nodes in $\tilde{\mathcal{T}}$. The main task of the fusion center is to decide whether the primary transmitter is in the ON or OFF state. We assume that all secondary nodes use identical energy detectors. Since the nodes in $\mathcal{T}$ are expected to be located relatively close to each other, the distributions of received power at these nodes are assumed to be identical and correlated.

Temporal spectrum sensing can be formulated as a binary hypothesis testing problem in which the fusion center determines whether or not the current mean received power is higher than the received power when the primary transmitter is in the OFF state [8]. We define two hypotheses: $H_{0}$ is the hypothesis that the primary is $\mathrm{ON}$ and located close to the secondary nodes, i.e., no spectrum hole exists, and $H_{1}$ is the hypothesis that the primary is OFF or far away, i.e., a spectrum hole exists. Thus, under $H_{1}$, a secondary node could reuse the frequency channel without causing interference to the primary system. Node $i \in \mathcal{T}$ performs temporal sensing by computing an observation $Y_{i}$, obtained by subtracting an estimate of the sum of the noise and interference power from the received power.

Let $\mathbf{Y}=\left(Y_{i}: i \in \tilde{\mathcal{T}}\right)$ denote the vector of observations at a given observation epoch. The hypothesis testing problem can then be formulated as follows:

$$
\begin{aligned}
& H_{0}: \mathbf{Y} \sim \mathcal{N}(\alpha \mathbf{1}, \mathbf{\Sigma}), \\
& H_{1}: \mathbf{Y} \sim \mathcal{N}\left(\mathbf{0}, \sigma_{0}^{2} \mathbf{I}\right),
\end{aligned}
$$

with $\alpha \geq \mu$, where $\mu \triangleq E\left[10 \log _{10}(1+\mathrm{SNR})\right][\mathrm{dB}]$, and SNR is the signal-to-noise ratio at the secondary nodes at the largest distance from the primary user or, equivalently, the smallest mean received signal-to-noise ratio when the primary is ON. In (4) and (5), $\mathcal{N}(\mathbf{v}, \boldsymbol{\Sigma})$ denotes the multivariate Gaussian distribution with mean vector $\mathbf{v}$ and covariance matrix $\boldsymbol{\Sigma}$ and $\sigma_{0}^{2}$ is the variance of the noise power under $H_{1}$. The symbols $\mathbf{0}$ and $\mathbf{1}$ denote vectors of all zeros and ones, respectively, and $\mathbf{I}$ is the identity matrix of appropriate dimension. The $(i, j)$ element of the covariance matrix $\boldsymbol{\Sigma}$ is given by $\Sigma_{i j}=$ $\sigma_{1}^{2} \rho^{d_{i j} / D_{c}}$ where $d_{i j}$ is the distance between nodes $i$ and $j$ in meters, $\sigma_{1}^{2}$ is the variance of the noise power under $H_{1}$, and $\rho$ is the correlation coefficient between secondary nodes separated by a reference correlation distance $D_{c}$ in meters. The parameter $\alpha$ represents the mean power observed under $H_{0}$.

The probability of temporal interference with the primary transmitter is equivalent to the false alarm probability $p_{f}=$ $P_{0}\left(\delta=H_{1}\right)$, where $\delta$ is the decision rule used by the fusion center and $P_{0}(\cdot)$ is the probability measure under $H_{0}$. In general, the temporal interference probability $P_{0}\left(\delta=H_{1}\right)$ does not necessarily equal the spatial sensing interference probability $P_{s}(a)$ given in (3). The temporal sensing system is designed such that the probability of temporal interference is less than or equal to a pre-specified value $\kappa$ :

$$
p_{f}=P_{0}\left(\delta=H_{1}\right) \leq \kappa .
$$

The constraint (6) must be satisfied for all values of $\alpha \geq \mu$ in (5). Since the prior information about the distribution of the mean power $\alpha$ is unknown, the composite binary hypothesis testing problem given by (4) and (5) is designed under a robust and universally most powerful detection framework [12]. In other words, the system is designed such that (6) is satisfied for the least favorable value of $\alpha$, i.e., $\alpha=\mu$ [8]. This results in a simple Neyman-Pearson hypothesis testing problem:

$$
\begin{aligned}
& H_{0}: \mathbf{Y} \sim \mathcal{N}(\mu \mathbf{1}, \mathbf{\Sigma}), \\
& H_{1}: \mathbf{Y} \sim \mathcal{N}\left(\mathbf{0}, \sigma_{0}^{2} \mathbf{I}\right) .
\end{aligned}
$$

The final decision $\delta$ is made at the fusion center, which has access to only binary-value decisions made individually by the secondary nodes based on the observation vector $\mathbf{Y}$. We denote by $U_{i}$ the individual decision made by the $i$ th temporal sensing secondary node, based on the observation $Y_{i}$. Correspondingly, $\mathbf{U}=\left(U_{i}: i \in \tilde{\mathcal{T}}\right)$ denotes the vector of $0-1$ hard decisions made by the secondary nodes in $\tilde{\mathcal{T}}$.

Let

$$
L\left(Y_{i}\right) \triangleq \frac{p_{1}\left(Y_{i}\right)}{p_{0}\left(Y_{i}\right)}
$$

denote the likelihood ratio of the observation at node $i \in$ $\tilde{\mathcal{T}}$, where $p_{0}(\cdot)$ and $p_{1}(\cdot)$ denote, respectively, the posterior distributions under hypotheses $H_{0}$ and $H_{1}$, respectively. Then the optimal decision at node $i$ can be represented as $U_{i}=$ $I_{\left\{\ln L\left(Y_{i}\right)>\tau\right\}}$, where $I_{A}$ denotes the indicator function of the set $A$. A secondary node decides $H_{1}$ if $U_{i}=1$ and otherwise decides $H_{0}$. The threshold $\tau$ is chosen to ensure that (6) is 
satisfied. The fusion center makes a final decision based on the decision bit vector $\mathbf{U}$.

Under the so-called counting rule, the final decision is made by comparing the sum $\sum_{i \in \tilde{\mathcal{T}}} U_{i}$ to a decision threshold. If the sum $\sum_{i \in \tilde{\mathcal{T}}} U_{i}$ is greater than the threshold, the fusion center decides $H_{1}$ and otherwise decides $H_{0}$. The value of this threshold is obtained through simulation [8]. When the observations across all of the nodes are independent and identically distributed under both hypotheses, the counting rule detector is optimal, since the joint likelihood ratio of the bits is a function only type of the number of ones in the received bit vector $\mathbf{U}$. The counting rule detector is also efficient when the correlations between the individual observations $Y_{i}$ are relatively small.

When the observations at the secondary nodes are correlated, the Linear Quadratic (LQ) detector yields a significant performance gain over the counting rule detector, while still using only partial statistical knowledge about the correlated decision variables [8]. The LQ detector is based on the generalized signal-to-noise ratio or deflection criterion, and makes use of fourth-order statistics under $H_{1}$ and second order statistics under $H_{0}$. We consider a fusion rule based on a class of LQ detectors that compare a linear quadratic function of decision vector to a threshold. The optimal LQ detector is derived in [9] for an arbitrary noise probability distribution with finite fourth order moments. When the observations at the secondary nodes are correlated, the LQ detector provides a simple fusion rule that yields significant performance gain over the Counting Rule while still using only partial statistical knowledge about the correlated decision variables [8].

\section{Joint Spatial-Temporal Spectrum Sensing}

The basic idea of joint spatial-temporal sensing as follows. A group of secondary nodes cooperatively localizes the primary transmitter, e.g., using signal strength observations [4]. Concurrently, a (possibly different) set of secondary nodes performs temporal spectrum sensing using knowledge of the estimated location and transmit power of the primary transmitter from the spatial sensing process. By performing both spatial and temporal sensing, a group of secondary nodes acquires sufficient knowledge to exploit the presence of both spatial and temporal spectrum holes. In the remainder of this section, we discuss a model for joint spatial-temporal sensing, a heuristic for intelligently node selection for temporal sensing, and capacity expressions for the temporal sensing, spatial sensing, and joint spatial-temporal sensing schemes.

\section{A. Model}

When the primary transmitter is $\mathrm{ON}$, transmitter $i$ transmits with power equal to its estimated maximum interference-free transmit power $\operatorname{MIFTP}_{i}$. Otherwise, when it is OFF, a given secondary user can transmit with power up to a maximum level $P_{m}$. We assume that the secondary users can coordinate among themselves by means of a suitable medium access control (MAC) protocol. Secondary receivers are affected by both large-scale and small-scale fading. The small-scale fading is modeled as Rayleigh block fading where the fading coefficient
$H_{i i}$ is constant over $N_{u}$ time slots, with $N_{u}$ being the number of transmitter-receiver pairs involved in communications. The shadow fading is modeled by a lognormally distributed random variable [13].

When a temporal spectrum hole occurs, i.e., when the primary transmitter is OFF, a given secondary node can transmit with power up to a maximum level $P_{m}$. On the other hand, when the primary transmitter is $\mathrm{ON}$, the secondary node can still transmit, but in this case, its transmit power will be limited to its MIFTP with respect to the primary transmitter. The MIFTP estimated by the secondary node depends on the locations of the secondary node and the primary transmitter, as well as the power of the primary transmitter. The spatial information associated with the primary transmitter must be estimated during the ON state of the primary transmitter. At the same time, the spatial information concerning the primary transmitter can be used to improve the performance of temporal sensing. The availability of more accurate spatial information can improve the accuracy of temporal sensing, which in turn can improve the accuracy of the estimated spatial information. The simulation results presented in Section $\mathrm{V}$ demonstrate that a significant performance gain can be achieved by joint spatial-temporal sensing relative to pure spatial sensing and pure temporal sensing.

\section{B. Node Selection for Temporal Sensing}

In joint spatial-temporal sensing, the secondary nodes collaboratively perform both spatial and temporal sensing. The primary transmitter parameters estimated via spatial sensing are used to improve the accuracy of temporal sensing. Using the estimated location of the primary transmitter, the fusion center for detecting temporal spectrum holes can intelligently choose a subset of the observation data from the secondary nodes so as to optimize detection performance. We propose two criteria for node selection: (1) minimum distance from the primary transmitter; (2) minimum correlation values between pairs of signal strength observations.

Let $\mathcal{T}$ denote the set of secondary nodes involved in temporal sensing. Then the fusion center fuses the individual decisions from the "best" subset $\tilde{\mathcal{T}}$ of $\tilde{T}$ nodes from the $T=|\mathcal{T}|$ nodes in the set $\mathcal{T}$ based on one of the two criteria. We assume that the fusion center has knowledge of the approximate locations of the nodes in $\mathcal{T}$. In practice, the nodes in $\mathcal{T}$ could send location updates to the fusion center at regular intervals. We remark that the time-scale for location updates would be much larger than that of decision-making for temporal spectrum holes. With knowledge of the locations of the nodes in $\mathcal{T}$, the fusion center can achieve the first criterion straightforwardly: Simply let $\tilde{\mathcal{T}}$ be a subset consisting of the $\tilde{T}$ secondary nodes in $\mathcal{T}$ that are closest to the primary transmitter $p$.

The second criterion is generally more difficult to achieve. Algorithm 1 is a heuristic that attempts to choose a subset of nodes such that pairs of observations from these nodes have small correlations (cf. [1]). The heuristic initializes $\tilde{\mathcal{T}}$ to be the entire set $\mathcal{T}$ and then successively removes nodes from $\tilde{\mathcal{T}}$ until $|\tilde{\mathcal{T}}|=\tilde{T}$. At each step, the node chosen for removal 
from $\tilde{\mathcal{T}}$ is chosen by first finding the pair $(a, b)$ of nodes in $\tilde{\mathcal{T}}$ that are closest to each other. Then $a$ or $b$ is removed from $\tilde{\mathcal{T}}$ according as $a$ or $b$ is farther from the primary transmitter $p$, respectively. The heuristic of Algorithm 1 is applied in the simulation results discussed in Section V-B.

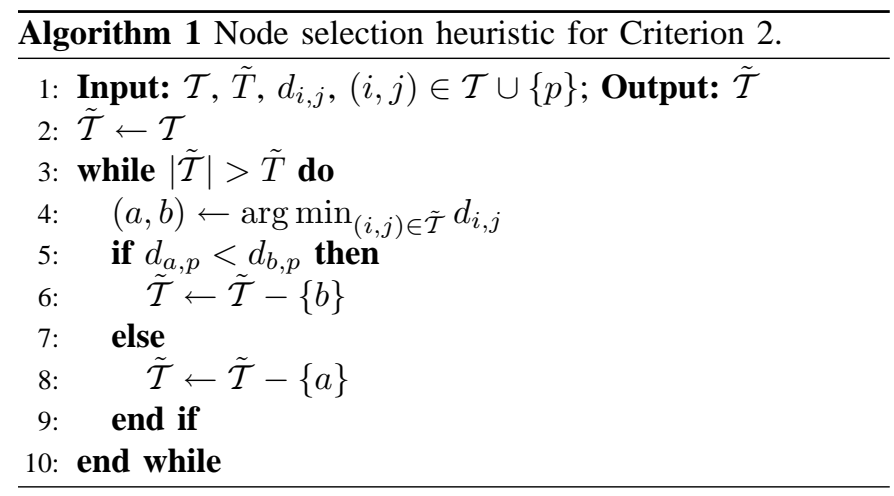

\section{Achievable capacity}

Next, we consider the achievable capacity of the proposed joint spatial-temporal sensing scheme relative to that of pure temporal sensing and pure spatial sensing. We adopt the narrowband spatial capacity model in [14] with the addition of shadow fading. Assume that $N_{u}$ pairs of secondary transmitters and receivers are placed within a circular region centered at the primary transmitter with radius equal to $R$. The location of receiver $i$ is assumed to be uniformly distributed over a circular strip bounded by two concentric circles centered at transmitter $i$, of radius $d_{\min }$ and radius $d_{\max }$, respectively. Under this assumption, the distance $D_{i i}$ has the following pdf (cf. [14]):

$$
f_{D_{i i}}(d)=\frac{2 d}{d_{\max }^{2}-d_{\min }^{2}}, \quad d \in\left[d_{\min }, d_{\max }\right],
$$

$i=1, \ldots, N_{u}$. In [14], the number of transmitter-receiver pairs, $N_{u}$, is assumed to be a Poisson random variable, but for the purposes of this discussion we will assume that $N_{u}$ is constant. We further assume a time division multiple access (TDMA) model wherein each frame contains $N_{u}$ time slots that are scheduled for user transmissions.

Under pure spatial sensing, transmitter $i$ can transmit to receiver $i$ with power level $\operatorname{MIFTP}_{i}$. Hence, the achievable capacity for the $i$ th transmitter-receiver pair is given by

$$
C_{\mathrm{S}, i}=B \cdot E\left\{\log _{2}\left(1+\frac{\operatorname{MIFTP}_{i}\left(D_{i i} / d_{0}\right)^{-n} W}{N_{0} B}\left|H_{i i}\right|^{2}\right)\right\},
$$

where the expectation $E[\cdot]$ is taken with respect to the transmitter-receiver distance $D_{i i}$, the shadowing noise $W$ and fading coefficients $H_{i i}$. As in [14], we assume that the channel gain between transmitter $i$ and receiver $j$ is normalized, i.e., $E\left\{\left|H_{i j}^{2}\right|\right\}=1$. Therefore, the average capacity under pure spatial sensing is given by $\bar{C}_{\mathrm{S}}=\frac{1}{N_{u}} \sum_{i=1}^{N_{u}} C_{\mathrm{S}, i}$.

Let $p_{\text {on }}$ and $p_{\text {off }}$ denote the probability that the primary transmitter is $\mathrm{ON}$ and the probability that it is OFF, respectively. Let $p_{d}=P_{1}\left(\delta=H_{1}\right)$ denote the probability of correct detection of a temporal spectrum hole, i.e., the probability that the fusion center correctly decides that the primary transmitter is OFF given that it is in fact in the OFF state. If the primary transmitter is OFF and the fusion center makes a correct detection decision, then secondary node $i$ can transmit with power up to a maximum level $P_{m}$. Hence, the achievable capacity under pure temporal sensing for the $i$ th transmitterreceiver pair is given by

$$
C_{\mathrm{T}, i}=p_{\mathrm{off}} p_{d} B \cdot E\left\{\log _{2}\left(1+\frac{P_{m}\left(D_{i i} / d_{0}\right)^{-n} W}{N_{0} B}\left|H_{i i}\right|^{2}\right)\right\} .
$$

Hence, the average capacity of pure temporal sensing scheme can be expressed as $\bar{C}_{\mathrm{T}}=\frac{1}{N_{u}} \sum_{i=1}^{N_{u}} C_{\mathrm{T}, i}$.

In joint spatial-temporal sensing, a given secondary node $i$ achieves the temporal sensing capacity $C_{\mathrm{T}, i}$ plus additional capacity due to spatial sensing when the primary transmitter is in the ON state. By combining (7) and (8), we can obtain the achievable capacity of joint spatial-temporal sensing as follows:

$$
C_{\mathrm{ST}, i}=C_{\mathrm{T}, i}+\left[p_{\text {off }}\left(1-p_{d}\right)+p_{\text {on }}(1-\kappa)\right] C_{\mathrm{S}, i},
$$

where $\kappa$ is the probability of temporal interference with the primary transmitter (cf. (6)). Here, we note that there is no spatial capacity gain when the secondary node collides temporally with the primary transmitter, i.e., when the secondary node decides that a temporal hole is present even though the primary transmitter is actually in the $\mathrm{ON}$ state. The average capacity under joint spatial-temporal sensing is then given by $\bar{C}_{\mathrm{ST}}=\frac{1}{N_{u}} \sum_{i=1}^{N_{u}} C_{\mathrm{ST}, i}$.

\section{Overhead}

The overhead of joint spatial-temporal sensing compared to pure temporal sensing consists of the additional computation carried out by the fusion center to select the subset of temporal sensing nodes. After the subset of temporal sensing nodes is determined by the fusion center, this set will remain unchanged until the fusion center selects a new subset. In general, the fusion center selects a new subset of temporal sensing nodes when the location of the primary transmitter changes. We assume that the time scale over which the primary transmitter changes its location is much larger than the time scale of its ON/OFF durations. Under this assumption, the extra overhead of joint spatial-temporal sensing compared to temporal sensing is not significant in practice. Compared to pure spatial sensing, the overhead of joint spatial-temporal sensing consists of the overhead of the temporal sensing process. The optimal design of the temporal sensing duration and the associated throughput of a cognitive radio has been studied in [15].

\section{TEMPORAL SENSING WITH MULTI-BIT FEEDBACK}

In the counting rule and LQ detectors, all the temporal sensing nodes send only a one-bit decision to the fusion center which fuses all the local hard decisions to arrive at a final decision. We propose an $m$-bit feedback approach for counting rule detector, whereby each node divides its observation region into $2^{m}$ quantization levels and sends an $m$-bit decision to fusion center. 


\section{A. Centralized Detector}

In centralized detection, a subset $\tilde{\mathcal{T}}$ of secondary nodes sends a set of soft observations $Y_{i}, i=1,2, \ldots,|\tilde{\mathcal{T}}|$ to the fusion center, where a joint likelihood ratio test on the entire vector $\mathbf{Y}$ is performed. The posterior pdfs are given by

$$
\begin{aligned}
& f_{\mathbf{Y}}\left(\mathbf{y} \mid H_{0}\right)=\frac{1}{(2 \pi)^{n / 2} \operatorname{det}\left(\Sigma^{1 / 2}\right)} e^{-\frac{1}{2}(\mathbf{y}-\alpha)^{*} \Sigma^{-1}(\mathbf{y}-\alpha)}, \\
& f_{\mathbf{Y}}\left(\mathbf{y} \mid H_{1}\right)=\frac{1}{(2 \pi)^{n / 2}} \exp \left(\sum_{i=1}^{|\tilde{\mathcal{T}}|} \frac{-y_{i}^{2}}{2 \sigma_{0}^{2}}\right)
\end{aligned}
$$

where $\mathrm{x}^{*}$ denotes the complex conjugate transpose of vector $\mathbf{x}$. Combining (10) and (11) we obtain the joint log likelihood ratio of the received vector at the fusion center as follows:

$$
\ln L(\mathbf{y})=\ln \left(\operatorname{det} \Sigma^{1 / 2}\right)-\sum_{i=1}^{|\tilde{\mathcal{T}}|} \frac{y_{i}^{2}}{2 \sigma_{0}^{2}}+\frac{(\mathbf{y}-\alpha)^{*} \Sigma^{-1}(\mathbf{y}-\alpha)}{2} .
$$

The fusion center compares the received log likelihood ratio with a threshold. The threshold is determined such that the false alarm probability is below a predetermined constant $\kappa$.

\section{B. Multi-level quantization}

At node $i$, the $\log$-likelihood ratio $\ln L\left(Y_{i}\right)$ of the observation $Y_{i}$ is computed. The decision rule at each node is specified as follows:

$$
U_{i}= \begin{cases}0, & \text { if } \ln L\left(Y_{i}\right) \leq t_{l} \\ \theta_{i}, & \text { if } t_{l}<\ln L\left(Y_{i}\right)<t_{u} \\ 1, & \text { if } \ln L\left(Y_{i}\right) \geq t_{u}\end{cases}
$$

where $0<\theta_{i}<1$ and the region $\left(t_{l}, t_{u}\right)$ is called the region of no confidence. If the log-likelihood ratio of node $i$ falls into this region, it transmits a soft decision $\theta_{i}$ to the fusion center. The other two complementary regions to $\left(t_{l}, t_{u}\right)$ are called confidence regions. For a given node $i$ the value of $\theta_{i}$ is quantized using a scalar quantizer $\mathcal{Q}_{i}$, which maps the input variable $\theta_{i}$ belonging to the interval $[0,1]$ into the output variable $\theta_{i j}, j=1,2, \ldots, q$ where $\theta_{i 1}=0$ if $\ln L\left(Y_{i}\right) \leq t_{l}$ and $\theta_{i q}=1$ if $\ln L\left(Y_{i}\right) \geq t_{u}$. The number of quantization levels, $q$, is constrained by the communication rate of the channel, $R_{i}$, $i \in \tilde{\mathcal{T}}$. If $m_{i}$ is the number of assigned bits, the communication rate satisfies $0 \leq 2^{m_{i}} \leq R_{i}, i \in \tilde{\mathcal{T}}$.

We consider a uniform quantizer [16] that divides the closed interval $[0,1]$ into $q$ quantization levels, where 0 and 1 are two of the levels. Hence, the open interval $(0,1)$ is divided into $q-2$ quantization levels with uniform step size $\psi=$ $1 /(q-2)$. If the value of the log-likelihood function falls within the $j$ th quantization interval $(j=2,3, \ldots, q-1)$ the quantized value is taken to be the middle of that interval. The transfer characteristic function of the quantizer can be specified as

$$
\theta_{i}= \begin{cases}\theta_{i 1}=0, & \text { if } \ln L\left(Y_{i}\right) \leq t_{1}, \\ \theta_{i j}, & \text { if } t_{j-1}<\ln L\left(Y_{i}\right)<t_{j}, j=2, \ldots, q-1, \\ \theta_{i q}=1, & \text { if } \ln L\left(Y_{i}\right) \geq t_{q-1},\end{cases}
$$

where

$$
\theta_{i j} \triangleq\left(\frac{2 j-1}{2}\right) \psi, \quad i=1,2, \ldots \tilde{T}, \quad j=2,3, \ldots, q,
$$

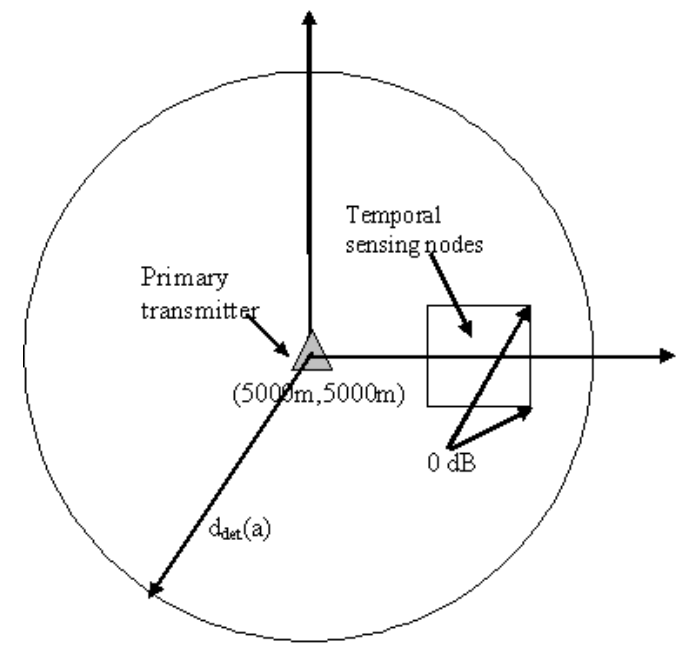

Fig. 1. Generation of secondary node locations.

$t_{1}=t_{l}$ and $t_{q-1}=t_{u}$. At the fusion center, the decision is made by comparing the sum of all received observations to a threshold $\tau$ :

$$
\delta= \begin{cases}H_{0}, & \text { if } \sum_{i=1}^{\tilde{T}} \theta_{i}<\tau \\ H_{1}, & \text { if } \sum_{i=1}^{\tilde{T}} \theta_{i}>\tau\end{cases}
$$

Since the detection metric is discrete-valued, randomization may be required to achieve equality in the interference probability constraint [12]. Randomization for the counting rule detector can be implemented by finding two thresholds

$$
\begin{aligned}
& \tau_{1}=\max \left\{\nu: P_{0}\left(\delta=H_{1} \mid \tau=\nu\right)<\kappa\right\}, \\
& \tau_{2}=\min \left\{\nu: P_{0}\left(\delta=H_{1} \mid \tau=\nu\right)>\kappa\right\},
\end{aligned}
$$

where $\kappa$ is a threshold that limits the probability of interference for temporal sensing (cf. (6)). Let $P_{\mathrm{t} 1}$ and $P_{\mathrm{t} 2}$ denote the interference probabilities obtained when using thresholds $\tau_{1}$ and $\tau_{2}$, respectively. The thresholds $\tau_{1}$ and $\tau_{2}$ are chosen with probabilities $1-p$ and $p$, respectively, where

$$
p=\frac{\kappa-P_{\mathrm{t} 1}}{P_{\mathrm{t} 2}-P_{\mathrm{t} 1}} .
$$

The average interference probability is then given by

$$
\kappa=p P_{\mathrm{t} 2}+(1-p) P_{\mathrm{t} 1} .
$$

When the observations are independent or the correlations between observations are small, the counting rule in (16) is optimum or near-optimum [16]. However, when the correlations among the observations are high, the counting rule detector (16) performs poorly.

\section{NUMERICAL RESULTS}

In this section, we compare the performance gain of the joint spatial-temporal sensing scheme with a pure temporal and spatial sensing schemes via simulation in various scenarios. In all scenarios, we assume that the transmit power, $s_{p}$, of the primary transmitter is unknown. Under joint spatial-temporal sensing, the secondary nodes collaboratively estimate both $s_{p}$ and the location of the primary transmitter. The following parameter settings are used in our simulation experiments: 


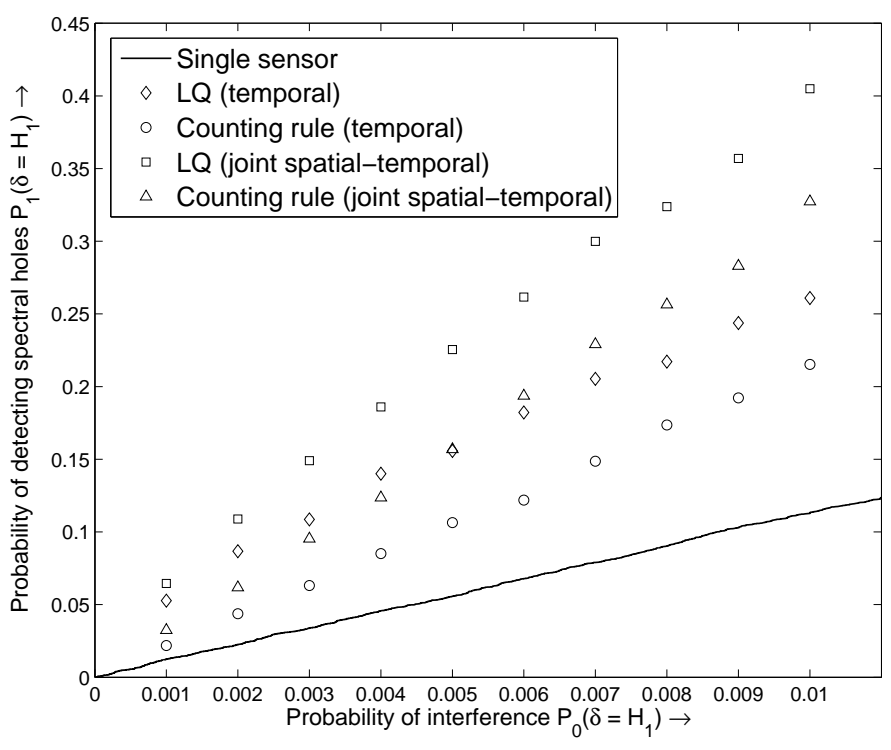

Fig. 2. Spatial-temporal sensing vs. temporal sensing with $\rho=0.6$.

- $r_{\min }=-30 \mathrm{dBm}, i_{\max }=-80 \mathrm{dBm}, \epsilon_{\mathrm{int}}=0.01$ and $\epsilon_{\mathrm{cov}}=0.05$

- $\sigma_{W}=4 \mathrm{~dB}, s_{p}=80 \mathrm{dBm}$, path loss factor $n=3$, $\sigma_{0}=1 \mathrm{~dB}$

- $\sigma_{1}=2.1 \mathrm{~dB}, \mu=3.4 \mathrm{~dB}$.

In the simulation experiments for achievable capacity, additional parameter settings are given as follows:

- $d_{0}=1 \mathrm{~m}, r_{\min }=10 \mathrm{~m}, r_{\max }=100 \mathrm{~m}, N_{u}=50$;

- $P_{m}=90 \mathrm{dBm}, p_{\text {off }}=p_{\text {on }}=0.5$ and $B=1 \mathrm{~Hz}$.

The primary transmitter is located at $L_{p}=(5,5) \mathrm{km}$. All secondary nodes are located in a disk of radius $R=100 \mathrm{~km}$. The MIFTP values of the secondary nodes range from zero to $60 \mathrm{dBm}$. The reference distance for temporal sensing nodes may be different from the reference distance in the disk centered at $L_{p}$ with radius $R$ because the temporal sensing nodes are located very far from the primary transmitter, i.e., where the received $\mathrm{SNR}=0 \mathrm{~dB}$. As shown in Fig. 1, the locations of $|\mathcal{S}|=20$ secondary nodes for spatial spectrum sensing are generated randomly with uniform distribution inside the circle centered at $L_{p}$ with radius equal to $d_{\text {det }}(a)$. All temporal sensing nodes are placed inside a square with the smallest possible mean received $\mathrm{SNR}=0 \mathrm{~dB}$. For the simulation results shown in Figs. 2-12, 95\% confidence intervals were computed, but they are omitted from the figures to maintain visual clarity of the plots.

\section{A. High correlation scenario}

In the first scenario, we assume the suburban environment correlation model in [17] with $d_{0}=1 \mathrm{~m}$, correlation coefficient $\rho=0.6$, and correlation distance $D_{c}=250 \mathrm{~m}$. We place 18 temporal sensing nodes inside the square area indicated in Fig. 1 with edge length equal to $D_{c} / 2=125 \mathrm{~m}$. Out of the 18 nodes, nine are placed in fixed locations along the edges of the square, with even spacing. In particular, assume that the bottom left corner of the square has coordinates $(0,0)$ and the length of an edge is 2 . Then the coordinates of the nine

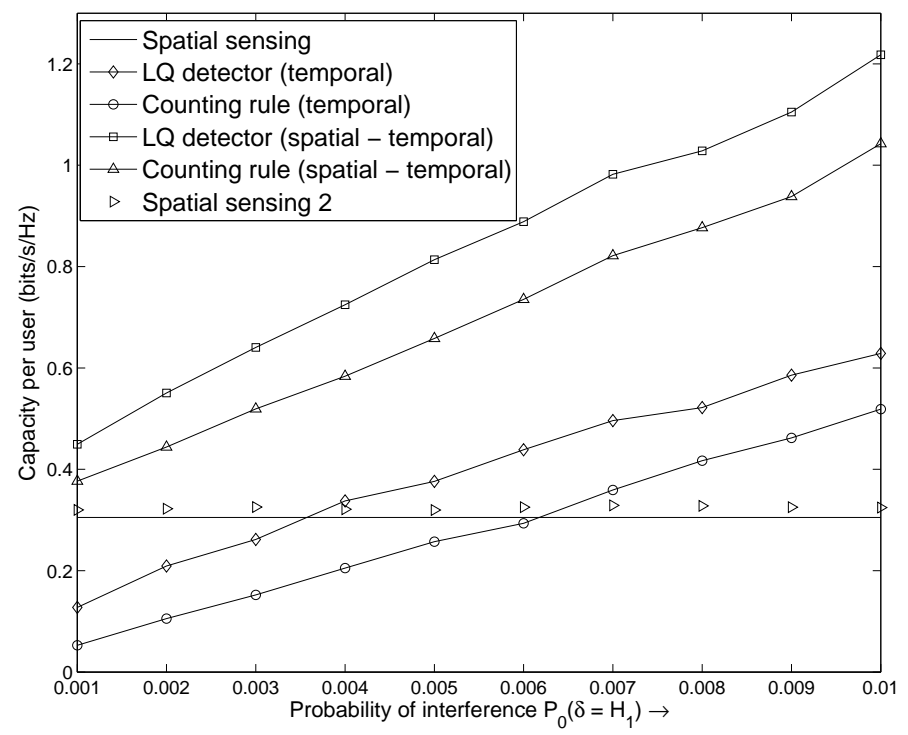

Fig. 3. Achievable capacity gain of joint spatial-temporal sensing, spatial sensing, and temporal sensing with $\rho=0.6$.

fixed locations are: $(0,0),(0,1),(0,2),(1,0),(2,0),(1,1)$, $(1,2),(2,1)$, and $(2,2)$. This placement of the nine nodes is the same as that used in [8]. The remaining 9 nodes are placed inside the square randomly according to a uniform distribution, i.e., the $\mathrm{x}$ and $\mathrm{y}$ coordinates for each of these nodes are drawn randomly from a uniform distribution on $[0,2]$. Because the nodes inside the square have different SNRs and the correlation $\rho$ is relatively large, the fusion center chooses the decisions from the nine nodes closest to the primary transmitter based on its estimated location.

Fig. 2 compares the detection performance of several temporal spectrum sensing schemes in this scenario. In both figures, the horizontal axis shows the probability of interference, $P_{0}\left(\delta=H_{1}\right)$. In Fig. 2, the performance of a single sensor is shown as the solid line. The performance of pure temporal sensing under the counting rule and the LQ detectors are shown with circles and diamonds, respectively. The LQ detector is seen to clearly outperform the counting rule, which confirms the results in [8]. Performance curves for joint spatial-temporal sensing using the counting rule and LQ detectors are shown with triangles and squares, respectively. The spatial-temporal sensing scheme is carried out using criterion 1 (see Section III). We see that the spatial-temporal LQ detector has the best performance over all values of $P_{0}(\delta=$ $H_{1}$ ). We also observe that the spatial-temporal counting rule detector performs worse than the temporal LQ detectors when $P_{0}\left(\delta=H_{1}\right)$ is small and better when $P_{0}\left(\delta=H_{1}\right)$ is larger; the crossover point is approximately 0.005 . Fig. 2 clearly shows the benefit of incorporating spatial information into temporal spectrum sensing.

Fig. 3 compares the average capacity of joint spatialtemporal sensing vs. pure temporal and pure spatial sensing. Clearly, the capacity achieved by the joint spatial-temporal scheme is significantly higher then that of the other schemes. 


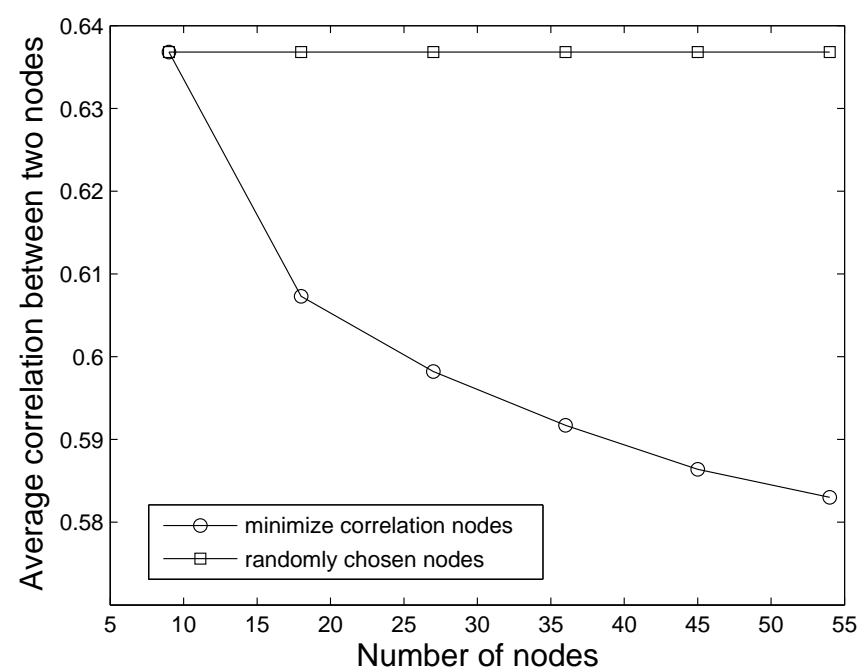

Fig. 4. Average correlation between the signal strength observations of two nodes over a subset of nodes selected by Algorithm 1 to minimize pairwise correlations.

In this figure, two performance curves associated with pure spatial sensing are shown. The curve labelled "spatial sensing" corresponds to the performance of a pure spatial sensing scheme when the primary transmitter is $\mathrm{ON}$ at all times. In this case, the secondary cannot benefit from the time intervals during which the primary transmitter may be OFF. The curve labelled "spatial sensing 2" shows the performance of a pure spatial sensing scheme operating in the presence of a primary transmitter that follows an ON-OFF pattern, but no additional temporal sensing information is employed. In this case, the MIFTP calculated by the secondary node varies over time due to the ON-OFF pattern of the primary transmitter, but the MIFTP cannot be determined accurately because signal strength measurements are taken by the secondary node regardless of whether the primary transmitter is ON or OFF. As a result, the MIFTP computed by a pure spatial sensing scheme at a given time may underestimate or overestimate the permissible transmit power. The latter case may result in harmful interference to primary users, while the former case may result in inefficient spectrum use. We observe from Fig. 3 that the capacity performance of "spatial sensing 2" is significantly poorer than that of the joint temporal-spatial sensing schemes, though slightly better than that of "spatial sensing." Note that the LQ-based detectors perform better than the counting rule based detectors, which one would expect, due to the relatively high correlation in this scenario.

\section{B. Moderate correlation scenario}

In the second simulation scenario, we set $d_{0}=100 \mathrm{~m}$, $\rho=0.3$, correlation distance $D_{c}=300 \mathrm{~m}$. All nodes in $\mathcal{T}$ have almost the same received SNR. In this scenario, we have $|\mathcal{T}|=18$ total nodes for temporal sensing, which are located randomly in the square shown in Fig. 1 according to a uniform distribution. A subset, $\tilde{\mathcal{T}}$ of $\tilde{T}=9$ nodes is chosen from the original set $\mathcal{T}$ according to one of the two criteria discussed in Section III. Fig. 5 compares the performance of the following four joint spatial-temporal detectors: (1) LQ detector under

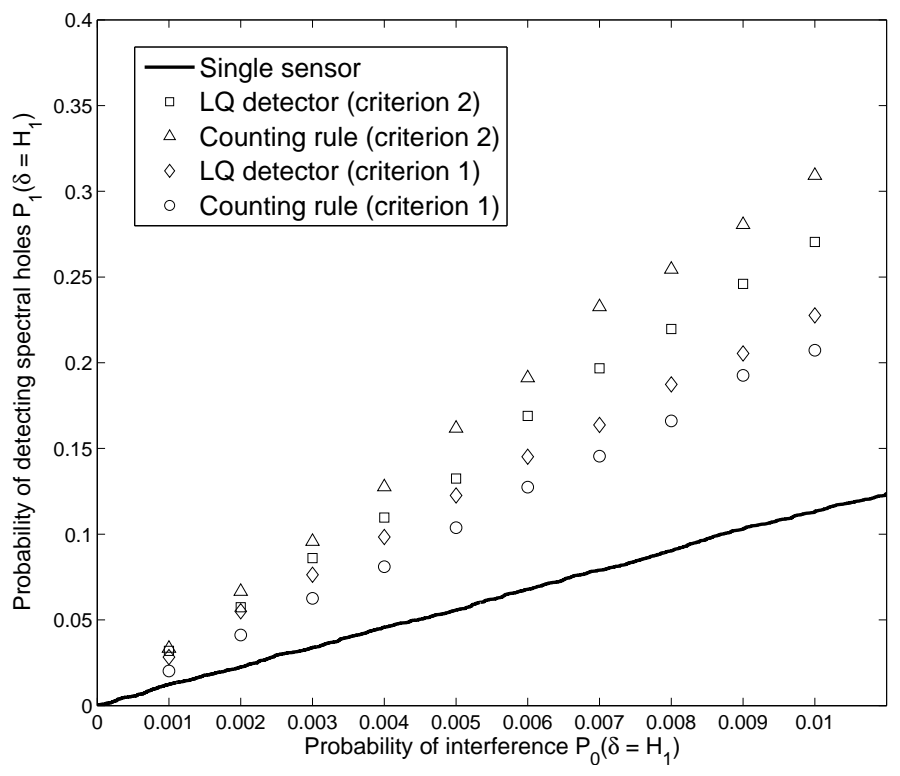

Fig. 5. Joint spatial-temporal sensing with different node selection criteria, $\rho=0.3$.

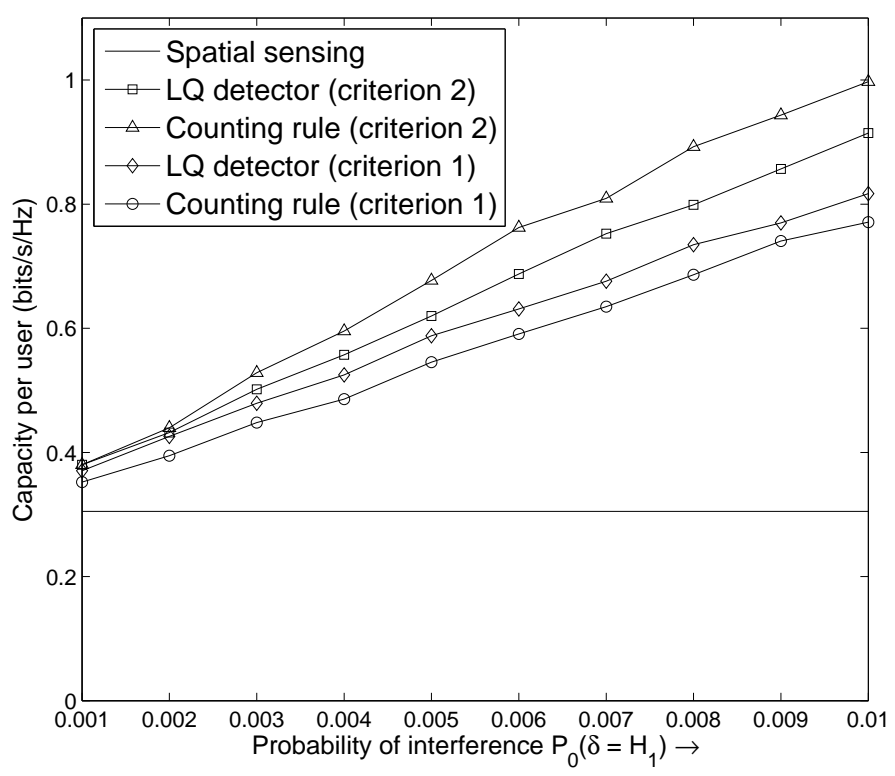

Fig. 6. Achievable capacity gain of joint spatial-temporal sensing, $\rho=0.3$

criterion 1; (2) Counting rule detector under criterion 1; (3) LQ detector under criterion 2; (4) Counting rule detector under criterion 2 .

In this scenario, the heuristic given as Algorithm 1 in Section III is used to implement criterion 2 approximately. Fig. 4 shows that the heuristic succeeds in reducing the average correlation between two nodes. As expected, the reduction in average correlation improves as the total number of secondary nodes increases. From Fig. 5, we observe that when the correlation is small and the received SNRs are similar, better performance is achieved with criterion 2, i.e., the nodes are selected using Algorithm 1. Under criterion 2, the counting rule detector outperforms the LQ detector because criterion 2 achieves low correlation among the observations, and when 


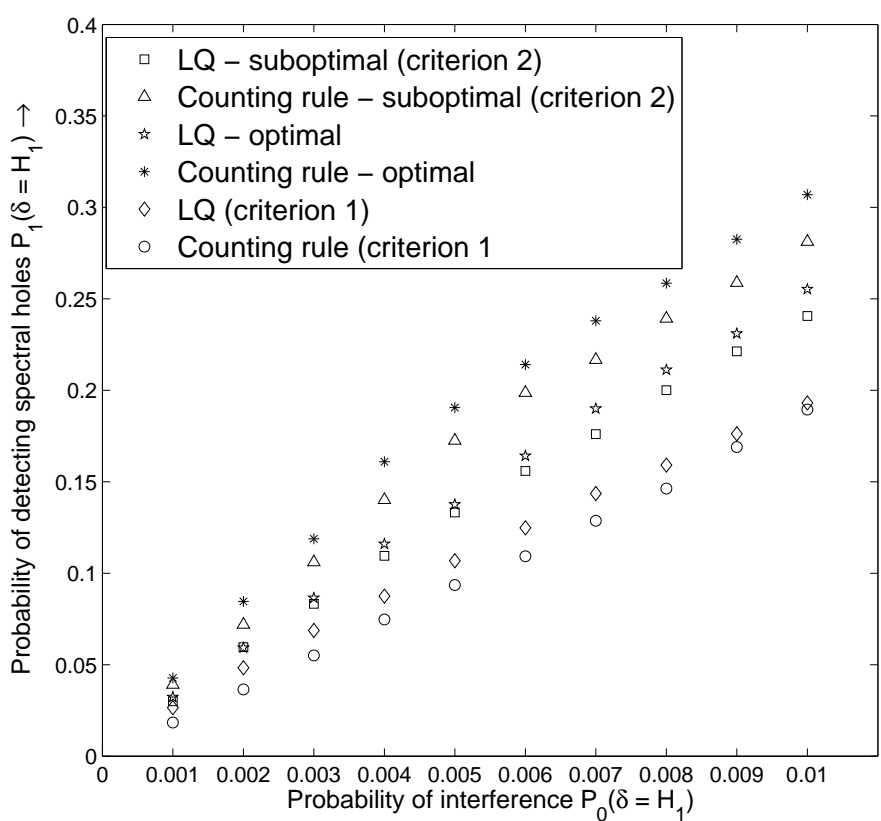

Fig. 7. Performance of optimum node selection vs. node selection based on Algorithm 1 with correlation parameter $\rho=0.3$.

the correlation is small the counting rule detector outperforms the LQ detector according to [8]. However, under criterion 1, the LQ detector still outperforms the counting rule detector because the correlation remains relatively high. In Fig. 6, capacity gains of the proposed scheme under both criteria are compared with that of a pure spatial sensing scheme. It can be seen that the use of criterion 2 achieves the largest capacity gain over spatial sensing.

In Fig. 7, we compare the performance of Algorithm 1 relative to an optimal selection of nodes. The optimal node set is found through simulation by searching over all possible node combinations. There are $\left(\begin{array}{l}|\mathcal{T}| \\ |\tilde{\mathcal{T}}|\end{array}\right)$ possible combinations, where $\mathcal{T}$ is the set of all nodes in the square area and $\tilde{\mathcal{T}}$ is the selected subset. In our simulations, we set $|\mathcal{T}|=9$ and $|\tilde{\mathcal{T}}|=5$. It can be seen that the performance of achieved by Algorithm 1 is quite close to that of an optimum node selection strategy. We remark that finding the optimum node subset is impractical when the number of combinations $\left(\begin{array}{l}|\mathcal{T}| \\ |\tilde{\mathcal{T}}|\end{array}\right)$ is large.

\section{Multi-bit feedback scheme}

In Fig. 8, we compare the performance of multi-bit counting rule temporal sensing in terms of detection probability and capacity vs. single-bit temporal sensing in a low correlation scenario with $\rho=0.2$. In this scenario, 9 nodes are uniformly distributed over the coverage area. In a region where the correlation is low, the multi-bit scheme significantly outperforms the counting rule detector. However, when the correlation parameter is high, the multi-bit scheme does not perform well, as shown in Fig. 9. This is because the detection rule at the fusion center is based on the counting rule, which performs well only when the correlation is small. The results of Fig. 9 also confirm that in a region with high correlation, the performance of the LQ detector is higher than that of counting

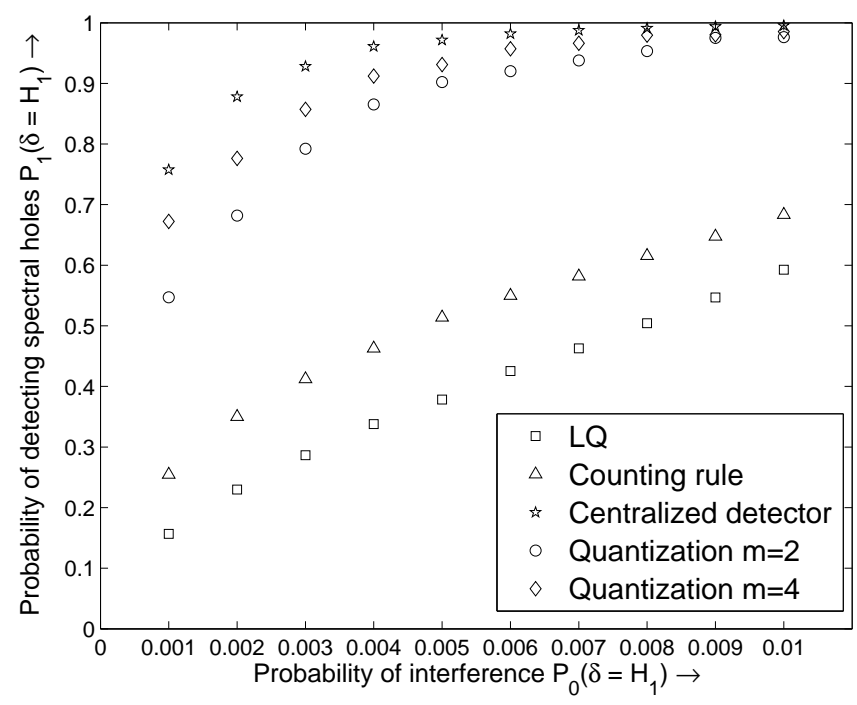

Fig. 8. Performance of multi-level quantization vs. other hard decision detection rules, $\rho=0.2$.

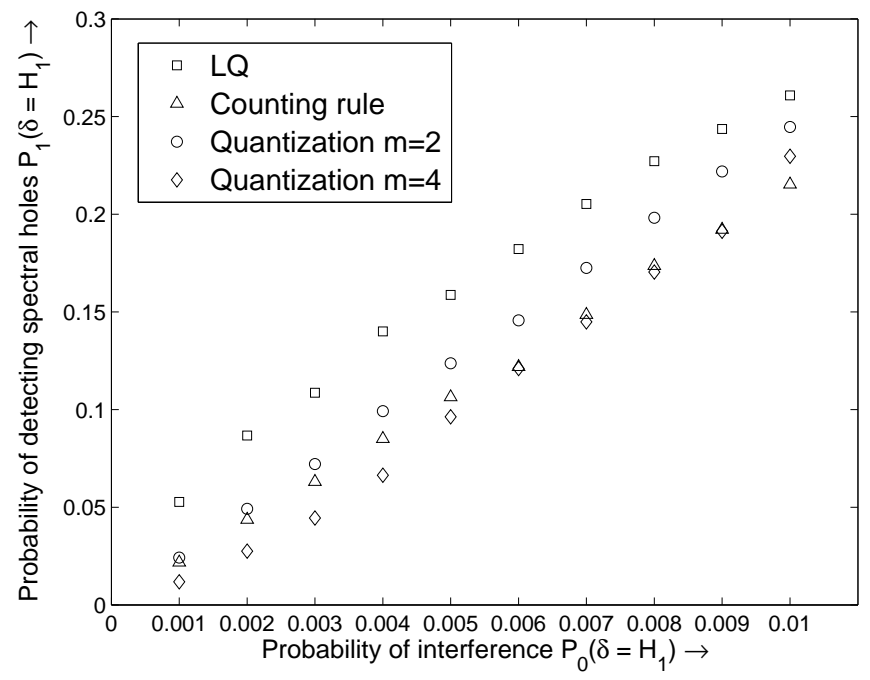

Fig. 9. Performance of multi-level quantization vs. other hard decision detection rules, $\rho=0.6$.

rule based detection schemes.

In Fig. 10, we compare the performance of the pure temporal LQ detector, the pure temporal counting rule detector, and pure temporal and joint spatial-temporal sensing with multilevel quantization $(m=2)$. The correlation parameters are set as follows: $\rho=0.3, d_{0}=100 \mathrm{~m}, D_{c}=300 \mathrm{~m}$. A total of $|\mathcal{T}|=18$ nodes perform temporal sensing and are located randomly in the square shown in Fig. 1 according to a uniform distribution. A subset, $\tilde{\mathcal{T}}$, of 9 nodes is chosen from the original set $\mathcal{T}$ using Algorithm 1, which seeks to minimize the correlation between nodes. In Fig. 11, we compare the capacity of the joint spatial-temporal sensing scheme with 2bit feedback vs. pure temporal and spatial sensing. We see that the capacity achieved by joint spatial-temporal sensing is significant higher than that of the pure temporal and spatial sensing schemes.

Fig. 12, shows the performance of the LQ detector, counting 


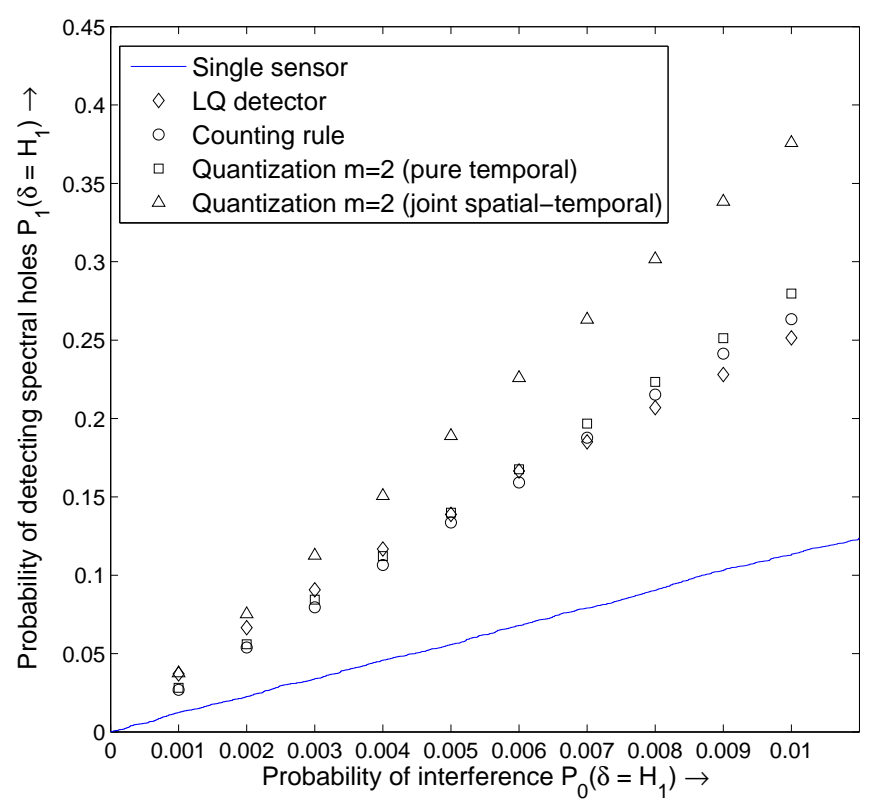

Fig. 10. Performance of multi-bit feedback detector vs. LQ and counting rule detectors, $\rho=0.3$.

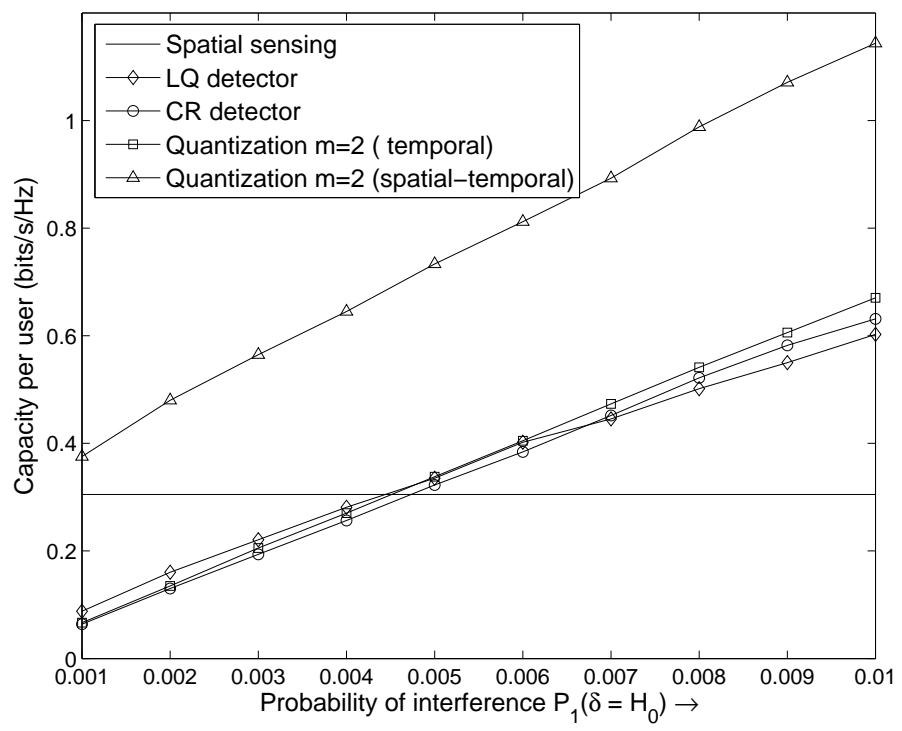

Fig. 11. Capacity gain of joint spatial-temporal sensing with 2-bit feedback.

rule detector, and multi-bit counting rule detector with $m=2$ and $m=4$ as a function of the correlation parameter $\rho$ and the interference probability constraint $P_{0}\left(\delta=H_{1}\right)=0.003$. Again, the LQ detector has the best performance when $\rho$ is large while the counting rule detector and multi-level counting rule detector perform well when $\rho$ is small. When the correlation is high, increasing the number of bits $m$ for multi-level feedback system does not improve the system performance appreciably.

Note that the performance curves for the counting rule based detectors decrease monotonically as functions of the correlation parameter $\rho$. On the other hand the performance curves for the LQ detector and the centralized detector are nonmonotonic: they first decrease and then increase as functions

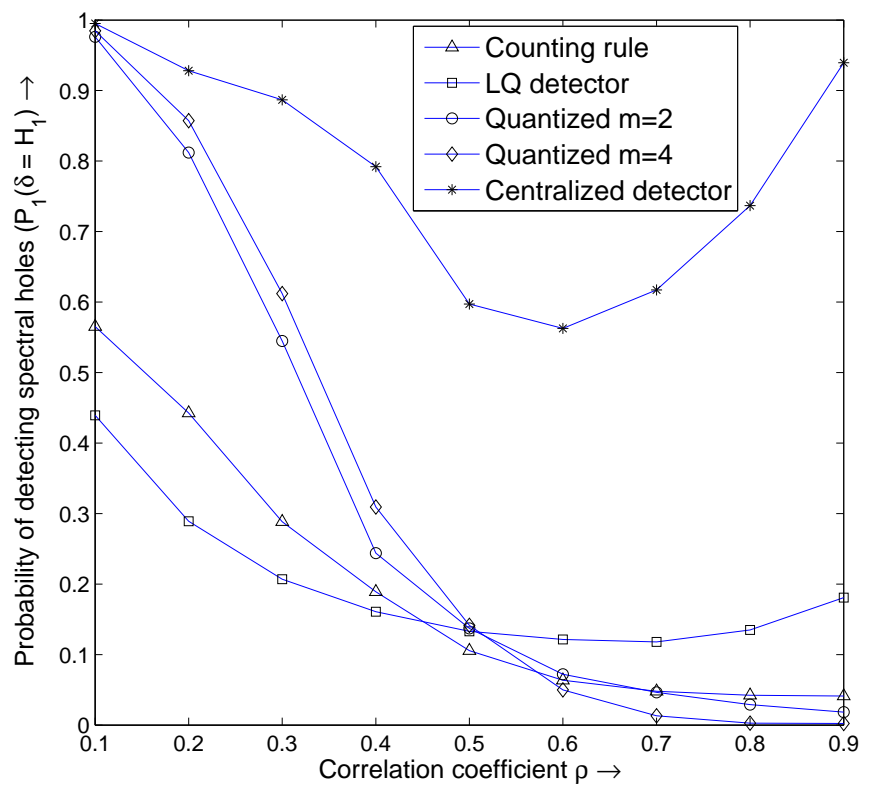

Fig. 12. Comparison of performance of LQ, Counting Rule and multibit feedback detectors as functions of correlation parameter $\rho$ with $P_{0}(\delta=$ $\left.H_{1}\right)=0.003$

of $\rho$. This can be explained in terms of two different features that can be exploited in the hypothesis testing problem given by (4) and (5). When the correlation parameter $\rho$ is small, the two hypotheses are distinguishable mainly by the mean values of the observations. In this case, the counting rule based detectors are expected to perform well. On the other hand, when $\rho$ is larger, the two hypotheses are more distinguishable in terms of second-order statistics, which the counting rule fails to capture. On the other hand, the LQ and centralized detectors exploit both features; hence, as we observe in the results, the performance curves first decrease and then increase as $\rho$ increases.

\section{CONCLUSION}

We proposed a joint spatial-temporal sensing scheme for opportunistic spectrum sharing in cognitive radio networks. The system model consists of a primary transmitter with unknown location and transmit power, which alternates between $\mathrm{ON}$ and OFF states, with respect to a given frequency channel. Spatial spectrum sensing is employed to estimate the maximum interference-free transmit power for a secondary node during an ON period. Estimates of the primary transmitter's location and transmit power obtained in the course of spatial sensing are used by a fusion center to select a subset of the secondary nodes to make a temporal sensing decision, i.e., a decision as to whether the primary is ON or OFF. Three distributed temporal sensing algorithms were considered: the counting rule detector, linear quadratic detector and counting rule with multi-bit feedback. By incorporating spatial information, we obtained joint spatial-temporal versions of these detectors. We derived the achievable capacity for pure temporal sensing, pure spatial sensing, and joint spatial-temporal sensing.

Our simulation results show that joint spatial-temporal sensing significantly outperform pure temporal sensing in terms 
of probability of spectrum hole detection and capacity gain. In this paper, we assumed only a single primary transmitter on a given frequency channel. In ongoing work, we are investigating joint spatial-temporal sensing in the presence of multiple cochannel transmitters.

\section{ACKNOWLEDGEMENT}

The authors would like to thank A. Nasif and J. Unnikrishnan for assistance in preparing the simulation codes and the anonymous reviewers for helpful comments that helped to improve the presentation of this paper.

\section{REFERENCES}

[1] T. Do and B. L. Mark, "Joint spatial-temporal spectrum sensing for cognitive radio networks," in Proc. 43rd Conf. on Information Systems and Sciences (CISS), Baltimore, MD, Mar. 2009.

[2] M. McHenry, "Frequency agile spectrum access technologies," in FCC Workshop on Cognitive Radio, May 2003.

[3] "Corvus: A cognitive radio approach for usage of virtual unlicensed spectrum," White Paper, Univ. California Berkeley, Jul. 2004.

[4] B. L. Mark and A. O. Nasif, "Estimation of maximum interference-free transmit power level for opportunistic spectrum access," IEEE Trans. Wireless Commun., vol. 8, no. 5, pp. 2505-2513, May 2009.

[5] — , "Estimation of interference-free transmit power for opportunistic spectrum access," in Proc. IEEE Wireless Comm. and Networking Conf. (WCNC'08), Las Vegas, NV, Apr. 2008.

[6] S. Mishra, A. Sahai, and R. W. Brodersen, "Cooperative sensing among cognitive radios," in Proc. IEEE Int. Conf. Communications, vol. 4, Istanbul, Jun. 2006, pp. 1658-1663.

[7] E. Visotsky, S. Kuffner, and R. Peterson, "On collaborative detection of TV transmissions in support of dynamic spectrum sharing," in Proc. 1st IEEE Int. Symp. New Frontier in Dynamic Spectrum Access Networks DySPAN, Nov. 2005, pp. 338-345.

[8] J. Unnikrishnan and V. Veeravalli, "Cooperative sensing for primary detection in cognitive radio," IEEE Journal Sel. Topics Signal Process., vol. 2, no. 1, pp. 18-27, Feb. 2008.

[9] B. Picinbono and P. Duvaut, "Optimal linear-quadratic systems for detection and estimation," IEEE Trans. Inf. Theory, vol. 34, no. 2, pp. 304-311, 1988.

[10] C. Perez-Vega and J. M. Zamanillo, "Path-loss model for broadcasting applications and outdoor communication systems in the VHF and UHF bands," IEEE Trans. Broadcasting, vol. 48, no. 2, pp. 91-96, Jun. 2002.

[11] B. L. Mark and A. O. Nasif, "Opportunistic spectrum sharing with multiple cochannel primary transmitters," IEEE Trans. Wireless Commun., vol. 8, no. 11, pp. 5702-5710, Nov. 2009.

[12] H. V. Poor, An Introduction to Signal Detection and Estimation, 2nd ed. NewYork, NY: Springer-Verlag, 1994.

[13] Z. Motamedi and M. R. Soleymani, "For better or worse: The impact of shadow fading on the capacity of large MIMO networks," in IEEE Global Telecommunication Conference (Globecom 2007), Nov. 2007, pp. $3200-3204$.

[14] D. Zhang, Z. Tian, and G. Wei, "Spatial capacity of narrowband vs. ultra-wideband cognitve radio systems," IEEE Trans. Wireless Commun., vol. 7, no. 11, pp. 4670-4680, 2008.

[15] Y.-C. Liang, Y. Zeng, E. C. Y. Peh, and A. T. Hoang, "Sensingthroughput tradeoff for cognitive radio networks," IEEE Trans. on Wireless Commun., vol. 7, no. 4, pp. 1326-1337, April 2008.

[16] A. M. Aziz, "A simple and efficient suboptimal multilevel quantization approach in geographically distributed sensor systems," Signal Processing, vol. 88, no. 7, pp. 1698-1714, Jan. 2008.

[17] M. Gudmundson, "Correlation model for shadow fading in mobile radio systems," IEEE Electron. Lett., vol. 27, no. 23, pp. 2145-2146, Nov. 1991.

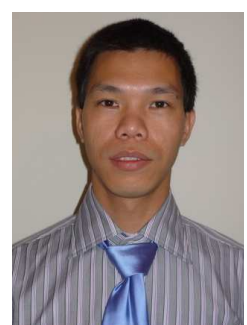

Tuan Do (S'09) graduated from Vietnam National University gifted high school and received the Bachelor of Engineering degree in Telecommunications Engineering from University of Communications and Transports, Hanoi, Vietnam in 1996 and 2001, respectively. As an undergraduate student, he won third prize in the National Math Olympic Competition and Second Prize in the University of Communication and Transport Computer Programming Competition. He received the Master of Science in Telecommunications and Computers from The George Washington University, Washington, DC in 2005. He has been working for Information System and Imaging Science Center at Georgetown University, Washington, DC since January 2008. Currently he is working toward the Ph.D. degree in Electrical and Computer Engineering at George Mason University, Fairfax, VA. His research interests are in wireless communications, network coding, and cooperative communications. He is a IEEE certified Wireless Communication Professional (WCP).

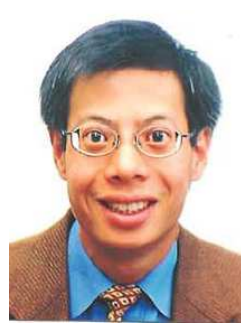

Brian L. Mark (M'91-SM'08) received the B.A.Sc. degree in Computer Engineering with an option in Mathematics from the University of Waterloo, Canada, in 1991 and the Ph.D. in Electrical Engineering from Princeton University, Princeton, NJ, in 1995 . He was a Research Staff Member at the C\&C Research Laboratories, NEC USA, from 1995 to 1999 . In 1999, he was on part-time leave from NEC as a visiting researcher at Ecole Nationale Supérieure des Télécommunications in Paris, France. In 2000, he joined the Dept. of Electrical and Computer Engineering at George Mason University, where he holds the rank of professor. His research interests lie in the design, modeling, and analysis of communication systems, computer systems, and communication networks. He served as an associate editor for IEEE Transactions on Vehicular Technology from 2006-2009. He was co-recipient of the best conference paper award for IEEE Infocom'97 and received a National Science Foundation CAREER Award in 2002. 